\title{
Magnetically Activated Stereoscopic Vision System for Laparoendoscopic Single-Site Surgery
}

\author{
Massimiliano Simi, Michele Silvestri, Carmela Cavallotti, Monica Vatteroni, Pietro Valdastri, Member, IEEE, \\ Arianna Menciassi, Member, IEEE, and Paolo Dario, Fellow, IEEE
}

\begin{abstract}
In this paper, the authors present an innovative vision platform for laparoendoscopic single-site (LESS) surgery based on a wired and magnetically activated 5-degrees-of-freedom robot with stereovision. The stereoscopic vision module, developed using two off-the-shelf cameras and a light emitting diodes lighting system, is mounted on the robot tip. An autostereoscopic screen is adopted to display the surgical scenario as an alternative to 3-D helmets or polarizing glasses. A rough position of the stereocamera can be determined along the abdominal wall by dragging the robot with a set of external permanent magnets (EPMs). Once the camera is set in the desired position, the EPMs provide fixation, while the internal mechanism allows fine tilt adjustment. Considering the deformable round shape of the insufflated abdomen wall and in order to replicate the precise roll motion usually provided by the endoscopist's hands, this prototype embeds an actuated mechanism that adjusts the stereocamera horizon and thus prevents any visual discomfort. Finally, the platform was preliminarily tested in vivo in a LESS scenario, demonstrating its advantages for eliminating potential conflicts with the operative tools and enabling the introduction of an additional instrument through the same access port used for stereoscopic vision.
\end{abstract}

Index Terms-Image sensors, medical robotics, robotic camera, stereo vision.

\section{INTRODUCTION}

$\mathbf{O}$ N-GOING medical research effort aims to reduce morbidity and is moving toward scarless surgery. Representing the latest advance in minimally invasive surgery, laparoendoscopic single-site (LESS) surgery allows significant improvement in this direction. The access technique for LESS involves the use either of access ports or of a series of 5-mm trocars side by side in the same incision, which is approximately $25-30 \mathrm{~mm}$ large and typically placed at the patient's umbilicus [1]. The

Manuscript received March 9, 2011; revised July 15, 2011 and November 4, 2011; accepted March 27, 2012. Date of publication May 25, 2012; date of current version January 18, 2013. Recommended by Technical Editor Z. Zhu. This work was supported in part by the European Commission within the framework of the ARAKNES European Project EU/IST-2008224565 .

M. Simi, M. Silvestri, C. Cavallotti, M. Vatteroni, A. Menciassi, and P. Dario are with The BioRobotics Institute, Scuola Superiore Sant'Anna, 56127 Pisa, Italy (e-mail: m.simi@sssup.it; m.silvestri@ sssup.it; c.cavallotti@ sssup.it; m.vatteroni@sssup.it; arianna@sssup.it; dario@sssup.it).

P. Valdastri is with the STORM Lab, Mechanical Engineering Department, Vanderbilt University, Nashville, TN 322-7311 USA (e-mail: p.valdastri@vanderbilt.edu).

Color versions of one or more of the figures in this paper are available online at http://ieeexplore.iee.org.

Digital Object Identifier 10.1109/TMECH.2012.2198830 technical feasibility of transumbilical-LESS has been clearly demonstrated for a wide range of surgical procedures using different access ports already available on the market [2], [3]. Despite promising to concretely improve traditional minimally invasive surgery, LESS still presents technical challenges that are far from being solved [4]. One of these challenges is limited triangulation and retraction of tissue due to the confinement of optics and working instruments to a single axis [5]. This severely hampers the field of view (FOV) of the surgical scenario and the manoeuvrability of the instruments. In addition, outside the patient's abdomen, the conflict between the endoscopist manoeuvring the camera, and the surgeon controlling the assistive and operative instruments, reduces the available workspace and may lead to unexpected movements during surgery. A series of articulating instruments are currently available on the market, specifically designed to accomplish a certain degree of triangulation [6]. The vision system commonly used in LESS surgery is a rigid extralong endoscope coupled with an extracorporeal video-camera [7]. The restricted motion of this kind of endoscope through the access port results in a limited endoscopic view.

Today, there is only one commercial endoscope purposely designed for LESS, i.e., the Endoeye, developed by Olympus. The Endoeye is a chip-on-the-tip 2-D 30 endoscope with 5-mm diameter and a flexible tip providing $100^{\circ} \mathrm{FOV}$ [8].

The Da Vinci robotic system (Intuitive Surgical Inc., Sunnyvale, CA) has also been proposed for LESS surgery (or Robotic-LESS) [9]. Besides providing easier articulation, motion scaling, and tremor filtration [10], the robot's main advantage is a fine 3-D vision that is the key to restoring depth cue, normally lacking since the introduction of laparoscopy. The drawbacks of the robotic system, however, regard the viewing console that completely isolates the surgeon from the surrounding environment and the significant external and internal encumbrance of the entire system, not specifically designed for LESS.

Hence, further developments in robotic platform designs and visualization systems are needed. A possible solution for addressing LESS open issues is represented by softly tethered miniaturized camera robots. These robotic cameras are not constrained by the entry incision, allowing the surgeon to place additional instruments safely and appropriately. Moreover, these systems provide additional camera angles that increase surgical visualization and improve orientation. The systems are positioned intra-abdominally and stabilized by suturing [11], by needle locking [12] or by external permanent magnets (EPMs) placed on the abdominal skin [13], in order to guarantee a large 
FOV and to leave the access port free for a different instrument. A number of magnetic anchoring and guidance system cameras are presented in [14]-[16]. In [17] and [18], camera robots are developed with two active internal degrees of freedom (DOFs) and magnetic anchoring. A stereoscopic robotic camera (2 DOFs: pan and tilt) is described in [19] and [20]. However, this device does not include a lighting system nor an anchoring system, and it is sutured on the abdominal wall during experimental validation.

Our goal is to improve the present technology by proposing an innovative magnetically activated stereocamera robot [21]. The design specifications for a novel stereoscopic vision platform based on a wired and magnetically activated robot are reported in Section II. The system overview and the details of the imaging system are reported in Sections III and IV, respectively, while Section V illustrates the robot design in terms of DOFs and activation method. Experimental results are reported in Section VI.

\section{DeSIGN SPECIFICATIONS}

The system requirements related to the development of a LESS camera robot are determined by medical considerations, physical constraints, and technical limitations.

Imaging system. Image quality in diagnosis and surgery always seems to be insufficient; VGA resolution, however, could be well suited for a first assessment of robotic prototypes [22]. Obviously, 3-D vision is preferred over 2-D vision; furthermore, in order to accomplish the typical operative tasks in LESS procedures and to avoid stereoscopic distortion, the vision system must guarantee correct 3-D viewing from $50 \mathrm{~mm}$ up to $150 \mathrm{~mm}$ in scene depth. Efficient and uniform illumination is also fundamental to guarantee sharp images without 3-D distortions. Illumination must exceed the minimum luminous intensity required for the cameras to be in proper working conditions in every spatial location without saturation. Finally, the visualization of the operative images must allow comfortable viewing (i.e., without glasses, helmets, or immersive console) to the highest number of operators and trainees.

Robotic system. The robot should have the highest number of DOFs in the smallest size to facilitate surgical mini-invasive procedures. In addition, it must fit the surgical single incision. Thus, considering the outer diameter of the umbilical ports ranging between 25 and $30 \mathrm{~mm}$ [23], a diameter of $30 \mathrm{~mm}$ can be set as maximum size for the robotic camera. Furthermore, the robot must avoid contact between the stereocamera and organs during surgical procedures. Considering that the operative field varies from person to person, since similar robotic camera prototypes [11], [17] have been used in extensive clinical testing, their upper length limit of $110 \mathrm{~mm}$ can be considered adequate. As regards the DOFs, the pan and tilt are necessary for pointing toward the different areas of the abdomen, whereas the roll is fundamental for restoring the right perception of the horizon, thus facilitating the procedure and avoiding any visual discomfort. In order to replicate the precise motion usually provided by the endoscopist's hands, a $>5 \mathrm{r} / \mathrm{min}$ speed and $<1^{\circ}$ resolution

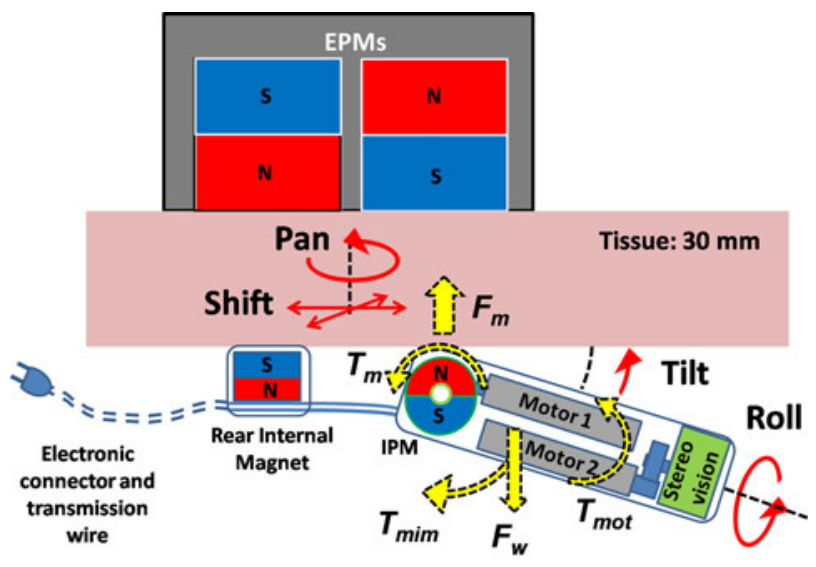

Fig. 1. Schematic view of the robotic system. The solid arrows represent the DOFs of the robot: three passive (Pan and Shift in two directions) and two active (Tilt and Roll). The dashed arrows represent all the forces and torques acting on the pivoting point of the IPM and considered in the model. The external permanent magnets (EPMs transmit the passive DOFs.)

are considered sufficient for each active DOF. Finally, possible sterilization of the entire device must be considered.

\section{SySTEM OVERVIEW}

Based on the aforementioned specifications, we designed a robotic 3-D vision system as schematically represented in Fig. 1. It incorporates a stereovision module at the tip, based on two cameras with two separate optical channels and a lighting system. A thin ( $2 \mathrm{~mm}$ in diameter) flexible wired connection to the external unit allows real-time video signal transmission, system control, and powering. Furthermore, it leaves the trocar free for the insertion of another tool, allowing effective retrieval from the abdomen in case of failure. A dedicated hardware is used for video data management. The signals from the cameras are sent to a frame synchronizing device that adapts the format to a 3-D display. Despite new shutter glasses TVs are cheap and userfriendly, an autostereoscopic monitor was adopted because it avoids isolating the observer from the surrounding environment and allows more than one observer to watch the surgical scene in 3-D at the same time without glasses. These features can improve the surgeon's performances if applied in the operating room [24]. On the other hand, autostereoscopic technology is still in its early stages and provides different solutions for achieving 3-D perception, each with specific strengths and weaknesses. Such a scattered scenario is the reason why previous assessment studies on autostereoscopic technologies present conflicting results [25]-[27]. In this study, an autostereoscopic display was adopted in order to provide an open console for the operator, without the need for any additional component, such as polarized glasses. This approach seems to be in line with the current trend of consumer electronics where large effort has been spent over the past years in autostereoscopic technology [28]-[30].

With regard to the system motion, three rough external DOFs, which are manually activated, correctly position and anchor the robot inside the abdomen, whereas other two motorized DOFs (Tilt and Roll) inside the robotic stereocamera accurately 
steer and orient the surgeon's point of view. The tilt DOF is actuated by the magnetic internal mechanism (MIM) [31], [32] and consists of a motor connected to an internal permanent magnet (IPM) by a set of gears. The device, immersed in an external magnetic field generated by EPMs, tends to maintain precise alignment defined by IPM polarization. When the motor is activated, the entire device rotates with it, while the IPM remains oriented according to the external field. Thanks to this operation principle, the MIM enables the device to tilt according to the surrounding tissue, without moving the EPMs. The second active DOF precisely adjusts the horizon of the stereo vision system. As regards horizon adjusting, it is worth mentioning that, while software image rotation is sufficient in case of a single camera, a hardware solution is required for a stereoscopic system in order to avoid reducing image resolution.

The head of the robot with two parallel cameras is connected to the motor by means of a set of cylindrical gears and can rotate with a span of $\pm 90^{\circ}$ thus guaranteeing horizon adjustment for all possible deviations. The two embedded motors are then controlled by a personal computer (PC) or by a specially developed pushbutton interface.

\section{IMAGING SYSTEM DESIGN}

\section{A. System Architecture}

The development of a stereoscopic imaging system includes two main parts: a device that acquires two 2-D images of the scene and a system that separates these images so that each observer's eye receives only one of them [33].

Among the several strategies available for acquiring stereoscopic image stream [7], [34], [35], the simplest method was adopted in this study, i.e., two separate optical channels were implemented by two adjacent cameras. The maximum size section for a square camera was set at $10 \mathrm{~mm} \times 10 \mathrm{~mm}$, so as not to exceed the maximum diameter of the LESS umbilical incision $(25 \mathrm{~mm})$. In addition, in order to balance the loss of brightness due to the dual-channel optics, a well-suited lighting system was required, as better detailed further on.

After a benchmark analysis, VGA CMOS color imagers, $8 \mathrm{~mm} \times 8 \mathrm{~mm} \times 9 \mathrm{~mm}$ in size, with pin-hole lens (Misumi Electronics Corporation, Taiwan) were chosen as the best tradeoff between image quality, low power consumption, embedded image processing tools, and low cost. These cameras have a FOV of about $60^{\circ}$ in horizontal and $52^{\circ}$ in vertical. The camera output is in NTSC (National Television System Committee) format, which can provide $400 \mathrm{TV}$ lines in resolution, with an image transfer rate of 60 frames-per-second (fps).

As display unit, a 19 in $(1280 \times 1024)$ autostereoscopic monitor (Pavonine Korea Inc., Korea) was used. This device employs the parallax barrier technology for 3-D visualization. The parallax barrier is an electro-optic panel with vertical, regularly spaced slits attached to the surface of a liquid crystal display. The slits are used to obscure parts of the two images coming from the cameras, thus spreading two separate 2-D images ahead of the monitor [36] (see Fig. 2). Consequently, the difference in the received right and left images, given by the horizontal separation of the cameras, is perceived by the observer's eyes

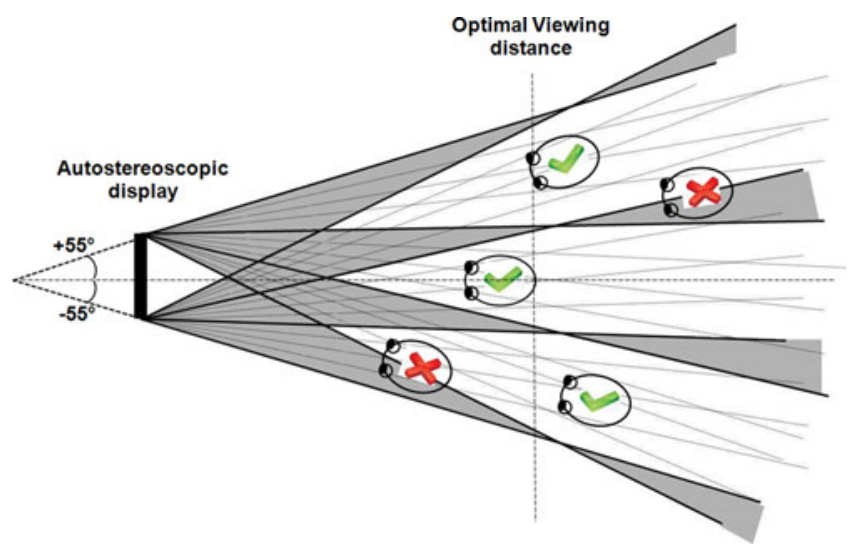

Fig. 2. Sweet-spots of the adopted autostereoscopic display, based on the parallax barrier technology.

without the need for helmets or glasses. The user's brain then fuses this difference, called disparity (measured in degrees or $\mathrm{mm}$ ), thus producing the perception of depth.

This screen has an optimal viewing distance of $80 \mathrm{~cm}$, with a tolerance, related to the observer's individual eye separation, of about $\pm 10 \mathrm{~cm}$, and guarantees a viewing angle of about $110^{\circ}$ with a correct stereoscopic zone, called sweet-spot, each about $2^{\circ}[37]$.

\section{B. System Dimensioning}

Imaging system dimensioning was based on the selected display and camera features and on the system specifications, in order to calculate the optimal distance between the cameras centers by means of geometrical models [38], [39]. Furthermore, the best configuration for the illumination system was theoretically studied by implementing a numerical model.

Two different camera setups are usually adopted in the state of the art [33]: the parallel and the toed-in setups. The first has a larger range of depth in which the disparity complies with the physiological limits, and also prevents peculiar stereoscopic distortions. The toed-in configuration instead has better 3-D rendering performance for a fixed target, where the background is less significant. The parallel configuration was chosen in this study because the target does not have a fixed and preknown position in the scene, and the background information is very useful for the surgeon. For this configuration, system dimensioning was carried out using the following equations [38], [39]:

$$
\left\{\begin{array}{l}
D_{\min }=M \cdot t \cdot f \cdot\left(\frac{1}{d_{0}}-\frac{1}{d_{\min }}\right) \\
D_{\max }=M \cdot t \cdot f \cdot\left(\frac{1}{d_{0}}-\frac{1}{d_{\max }}\right)
\end{array}\right.
$$

where $D_{\min }$ and $D_{\max }$ are, respectively, the minimum and the maximum allowed disparity values, $M$ is the value of frame magnification, $d_{\min }$ and $d_{\max }$ are the minimum and the maximum required scene depth values and $d_{0}$ is the zero-disparitydepth, $f$ the focal length, and $t$ the distance between the two camera centers. In the case of Misumi cameras, focal length is 


\section{LED Configuration: 1}

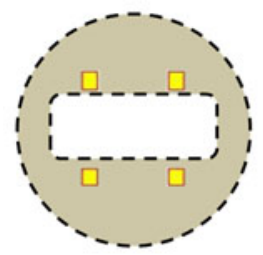

LED Configuration: 3

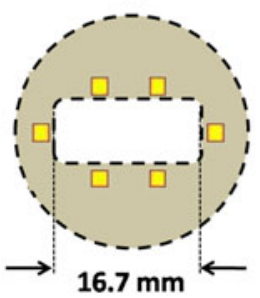

LED Configuration: 2

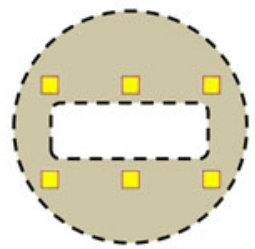

LED Configuration: 4

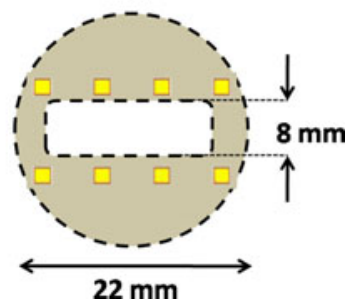

Fig. 3. Four LED configurations tested by the theoretical model. LEDs are shown inserted in the case $(22 \mathrm{~mm}$ in diameter) of the electronic illumination system that has a central hole for hosting the cameras.

$3.1 \mathrm{~mm}$, frame magnification is 93.75 (given by the ratio between sensor width and autostereoscopic monitor width). As defined in Section II, scene depth range is $50-150 \mathrm{~mm}$. The disparity limits are obtained from the following relations:

$$
\left\{\begin{array}{l}
D_{\min }=2 \cdot Z \cdot \tan \left(\frac{2 \cdot \arctan (e / 2 \cdot Z)+\mu}{2}\right)-e \\
D_{\max }=2 \cdot Z \cdot \tan \left(\frac{2 \cdot \arctan (e / 2 \cdot Z)-\mu}{2}\right)-e
\end{array}\right.
$$

where $Z$ is the optimal viewing distance from the autostereoscopic monitor $(80 \mathrm{~cm}), e$ is the typical human eye separation (65 mm) and $\mu= \pm 1.5^{\circ}$ is the physiological disparity limit [33]. Solving (1) using these disparity limits, the distance obtained between the two camera centers is $8.7 \mathrm{~mm}$, thus producing a maximum lateral size of both cameras of $8.7 \mathrm{~mm}$. This constraint agrees with the chosen cameras, whose width is $8 \mathrm{~mm}$.

Finally, the proposed system also required appropriate illumination, with light distribution in the scene as uniform as possible, in order to avoid artifacts in the 3-D viewing. As light source, white light emitting diodes (LEDs) (Nichia Corporation, Tokushima, Japan) were used, because of their high efficiency (1000 mcd of emitted power) and compact size $(2 \mathrm{~mm} \times$ $1.2 \mathrm{~mm} \times 1.3 \mathrm{~mm}$ ) [40]. Unfortunately, no models or guidelines for optimal distribution of the light sources for medical applications can be found in the literature. Therefore, an approximate mathematical model for light propagation was adopted. The implemented propagation law, based on the Lambert-Beer rule [41] and on the emission features of the LEDs, allows calculation of the luminous intensity associated with each light source for every spatial location in the workspace by applying the superposition effect. This model neglects scattering effects and reflection phenomena in order to hold down the computational load.

Four different LED configurations, shown in Fig. 3, were tested: one with four LEDs (configuration 1), two with six LEDs

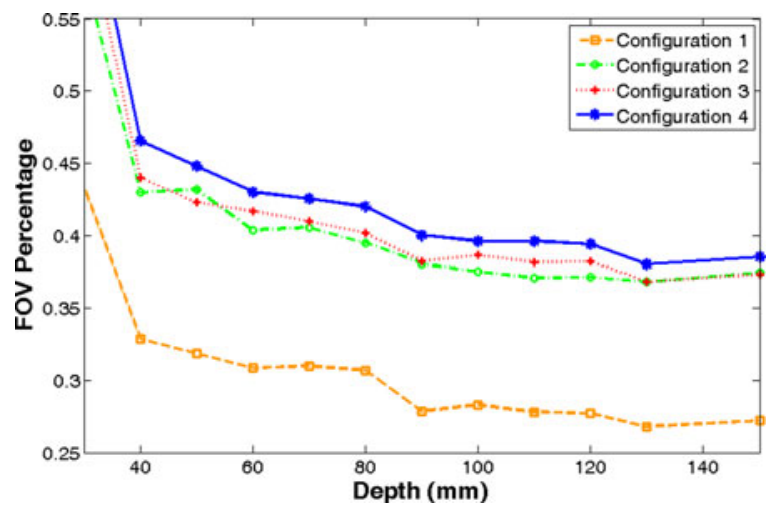

Fig. 4. Comparison between the LED configurations concerning the uniformity parameter, i.e., the percentage of the stereoscopic FOV having a CV less than $5 \%$. As the stereoscopic FOV size varies with depth, the uniformity parameter is also displayed along the scene depth.

using two different geometrical arrangements (configurations 2 and 3), and one with eight LEDs (configuration 4). A higher number of LEDs were not considered because of space constraints and in order to prevent sensor saturation. As illumination uniformity parameter, the percentage of the stereoscopic FOV having a coefficient of variation (CV) less than 5\% was evaluated for each configuration [41]. Moreover, the tested lighting configurations were requested to overcome minimum luminous intensity, equal to 0.2 lux, required for the cameras to operate in proper working conditions in every spatial location. Results from simulations are reported in Fig. 4. The eight LED configuration showed better performance in terms of illumination uniformity and guaranteed a minimum luminous intensity within the stereoscopic FOV much larger than the minimum value requested by the cameras (see Fig. 5). Therefore, the eight LED configuration, equally distributed above and below the camera, was selected.

The eight LEDs and necessary drivers were mounted on a printed circuit board, designed to surround the cameras at the same level of the sensors, thus avoiding unwanted reflections from the glass used to hermetically seal the device. Light source temperature ranged from $37^{\circ} \mathrm{C}$ to $40^{\circ} \mathrm{C}$, comparable with standard laparoscopic fiberoptic sources. The whole imaging system was finally $23 \mathrm{~mm}$ in diameter and $9 \mathrm{~mm}$ in depth (corresponding to the camera thickness), and weighed $3.8 \mathrm{~g}$.

\section{Robotic SySTEM DESIGN}

All robot parts were selected and designed considering stereovision module size, umbilical port diameter and the other mechanical requirements described earlier. In order to fabricate the chassis of the prototype with a stereo-lithographic rapid prototyping technique (3-D Printer Invision $\mathrm{Si}$ ), a total diameter of $25 \mathrm{~mm}$ was considered, which represents the limit guaranteeing solidity of the shell (minimum wall thickness of $1 \mathrm{~mm}$ ) and internal stability of all mechanical components. 


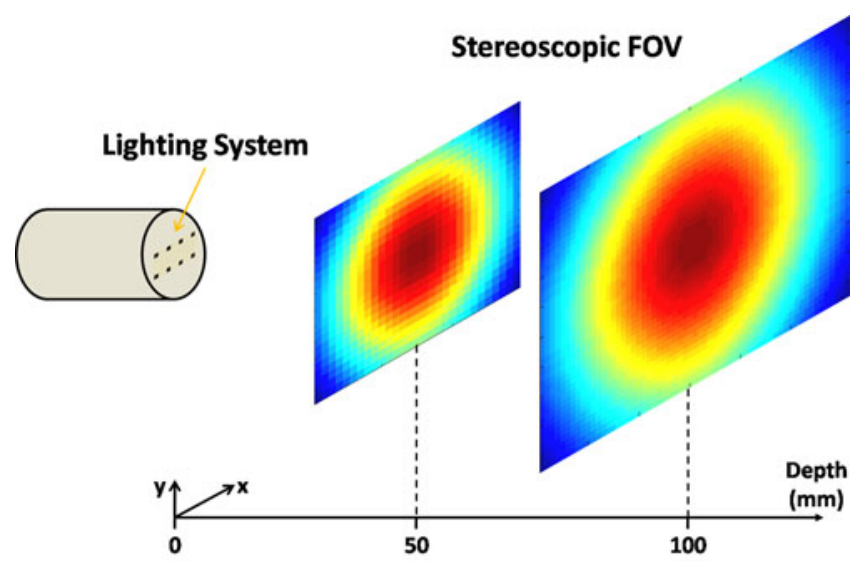

(a)

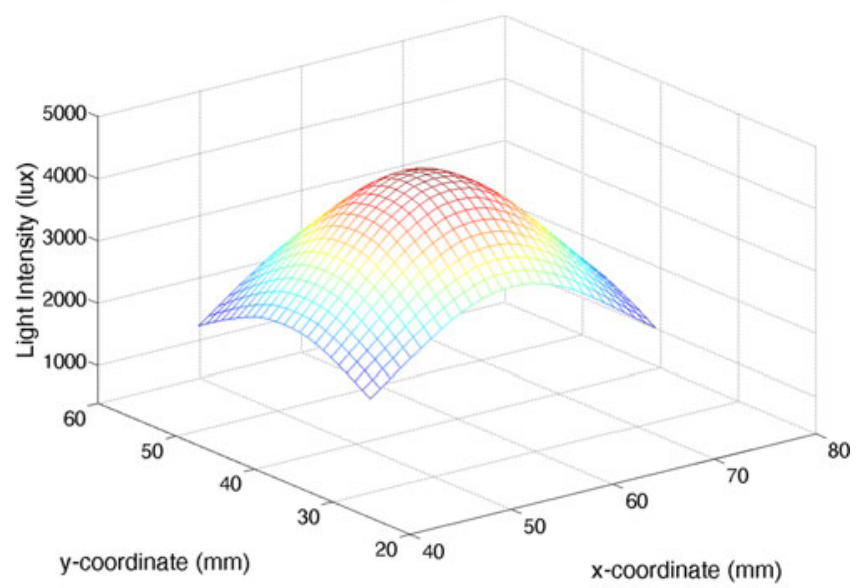

(b)

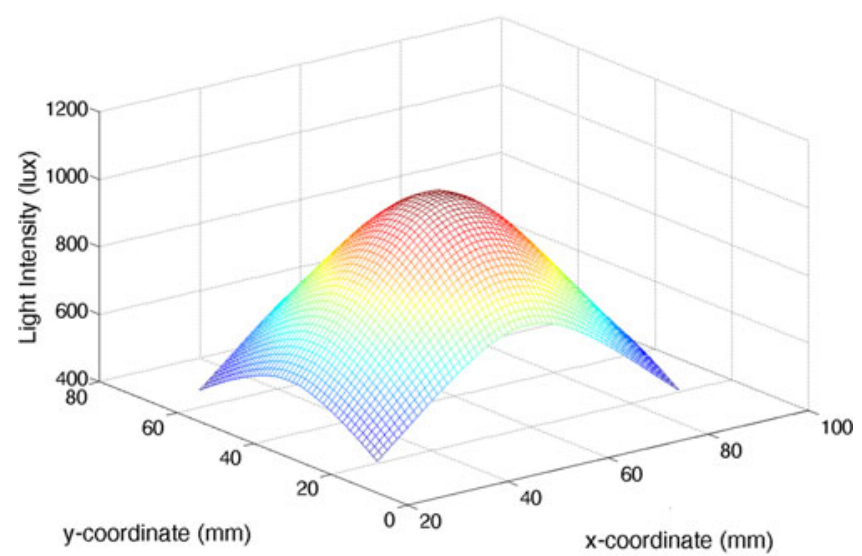

(c)

Fig. 5. (a) Schematic representation of the model geometry showing how the stereoscopic FOV was extrapolated and the origin of the coordinates. The theoretical lighting intensity distributions within the stereoscopic FOV at (b) $50 \mathrm{~mm}$ in depth and (c) $100 \mathrm{~mm}$ in depth. These distributions were obtained using LED configuration 4 . The $x$ - and $y$-axis are the coordinates, measured in $\mathrm{mm}$, implemented in the model. The $z$-axis is the light intensity measured in lux.

\section{A. IPM Selection}

As first step, the IPM features were defined to maximize magnetic attraction force and torque in the internal diameter $(23 \mathrm{~mm})$

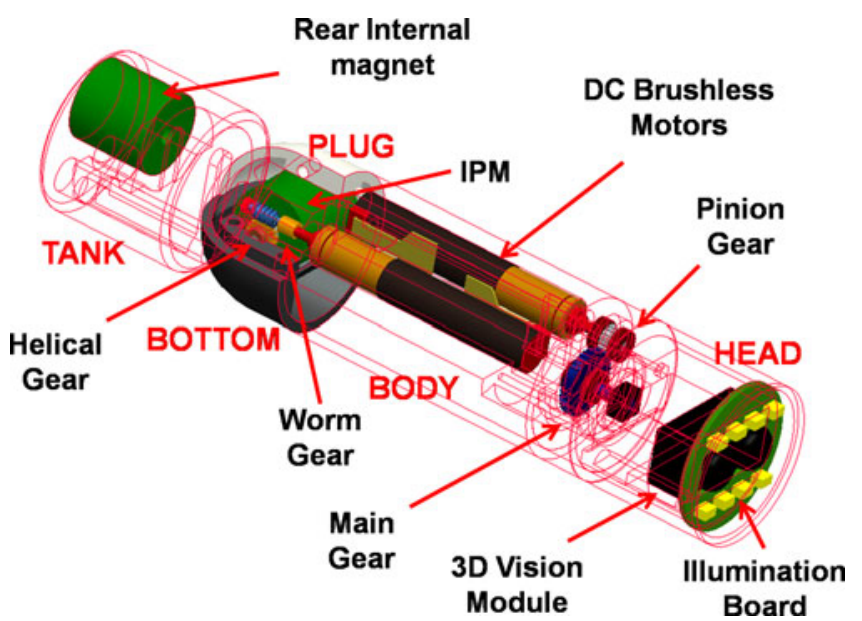

Fig. 6. 3-D design of the robotic stereocamera. The main embedded components of the device and the parts of the chassis are shown.

of the robot, also considering the mechanism configuration as represented in Fig. 6. A diametrically magnetized commercially available NdFeB N52 permanent magnet (KJ Magnetics, Jamison, PA) was selected. The permanent magnet has a ring shape, measures $12.7 \mathrm{~mm}$ in diameter and $12.7 \mathrm{~mm}$ in thickness, and has an axial hole of $3.2 \mathrm{~mm}$. Another ring magnet, with the same IPM features, was fixed at $3 \mathrm{~cm}$ from the bottom of the robot in a dedicated rear tank, which allows the robot to be coupled with two EPMs, thus stabilizing anchorage and providing the external pan and $x-y$ translation. The EPMs must be selected once the entire robot system has been designed and assembled in order to satisfy the technical requirements related to magnetic anchoring, external pan motion, and magnetic tilt.

\section{B. Motor Selection and Design of Mechanisms}

In order to satisfy the speed and resolution requirements of the two internal DOFs (tilt and roll), two Maxon EC6 DC brushless motors [42], with 221:1 planetary gearhead and inductive encoder were selected as the best commercially available tradeoff between resolution $\left(\ll 0.01^{\circ}\right)$, max speed $(136 \mathrm{r} / \mathrm{min})$, output torque (stall torque of $120 \mathrm{mNm}$ ), compactness $(6 \mathrm{~mm}$ in diameter and $35 \mathrm{~mm}$ in length), and simple control. Furthermore the nonbackdriveability of the two motors holds the robot in position without consuming power.

The negligible effect of high magnetic fields on the on-board motor output was experimentally verified as in [43]. No rotation speed variation was observed, even with the motor surrounded by $0.5-\mathrm{T}$ magnetic flux density.

The IPM of the MIM was connected to the motor by means of a bronze helical gear and a steel worm gear with transmission ratio and efficiency set at 0.056 and 0.415 , respectively. Regarding horizon adjustment, two ergal cylindrical gears (pinion and main gears) were designed with a transmission ratio of 0.4 and an efficiency of 0.85 . Considering half of the motor stall torque as the operative value, the torque $T_{\text {mot }}$ exerted on the IPM by the motor is $445 \mathrm{mNm}$, whereas $128 \mathrm{mNm}$ are transmitted to the robot head. Finally, adopting relations in [44] and considering 
TABLE I

GEAR FEATURES

\begin{tabular}{|l|l|l|l|l|}
\hline & $\begin{array}{l}\text { Pinion } \\
\text { Gear }\end{array}$ & $\begin{array}{l}\text { Main } \\
\text { Gear }\end{array}$ & $\begin{array}{l}\text { Worm } \\
\text { Gear }\end{array}$ & $\begin{array}{l}\text { Helical } \\
\text { Gear }\end{array}$ \\
\hline MODULUS & 0.25 & 0.25 & 0.25 & 0.25 \\
\hline $\begin{array}{l}\text { TEETH } \\
\text { NUMBER }\end{array}$ & 16 & 40 & 1 & 18 \\
\hline $\begin{array}{l}\text { PITCH } \\
\text { CIRCLE }\end{array}$ & $4 \mathrm{~mm}$ & $10 \mathrm{~mm}$ & $2.051 \mathrm{~mm}$ & $4.534 \mathrm{~mm}$ \\
\hline ADENDUM & $0.25 \mathrm{~mm}$ & $0.25 \mathrm{~mm}$ & 0.25 & $0.25 \mathrm{~mm}$ \\
\hline DEDENDUM & $0.3125 \mathrm{~mm}$ & $0.3125 \mathrm{~mm}$ & $0.3125 \mathrm{~mm}$ & $0.3125 \mathrm{~mm}$ \\
\hline $\begin{array}{l}\text { PRESSURE } \\
\text { ANGLE }\end{array}$ & $20^{\circ}$ & $20^{\circ}$ & $20^{\circ}$ & $20^{\circ}$ \\
\hline $\begin{array}{l}\text { HELIX } \\
\text { ANGLE }\end{array}$ & - & - & $7^{\circ}$ & $7^{\circ}$ \\
\hline
\end{tabular}

the motor features, the theoretical maximum speed and resolution can be derived as 7.6 and $53.3 \mathrm{r} / \mathrm{min}$ for the magnetic tilt motion and the horizon active rotation, respectively, with maximum resolution $<0.1^{\circ}$. All the gear features (worm, helical, pinion and main gears) are reported in Table I.

\section{Fabrication and Assembly}

Both pinion and main gears were fabricated using a 5-axis micro-CNC machining center (HSPC, KERN GmbH, Germany) as in [45], whereas the steel worm gear and the bronze helical gear were custom designed by the authors and fabricated by an external workshop. The gears were modified by Sink and Micro Wire Electro Discharge Machining (EDM) (Micro Sink, Sarix, Switzerland, and AP 200 L, Sodick, Japan, respectively) to provide proper couplings between connecting parts. In particular, the worm gear was cut at one end in order to obtain a rectangular groove, while a T-shaped hole was made in the helical gear to enable proper connection with the brass shaft as in [46]. The motor shafts were machined by Micro Wire EDM and fitted into the hole of dedicated brass bushes, whereas a brass holed plate was used to link the main gear to the robot head. All gear shafts were assembled on ball bearings, apart from the worm gear that was mounted on custom-made bushes in synthetic ruby with buffing surface to minimize the friction force and overall dimensions.

The plastic chassis is composed of five different parts (head, body, bottom, plug, and tank) that can be easily fitted together. The head, where the cameras are fixed, can rotate along the cylindrical axis with a span of $\pm 90^{\circ}$. The body, where the head and the bottom parts can be fixed, has two $6 \mathrm{~mm}$-diameter holes for the motor, two rectangular grooves for the motor connectors and a slot for the IPM. Additionally, a dedicated half-moon hole is obtained along the body that permits rotation of the cables from the cameras with the robot head. The bottom and the plug define the end part of the robot where the IPM is placed, whereas the tank embeds the second magnet for the active external pan/translation motions. Once assembled, the entire stereovision robot is $25 \mathrm{~mm}$ in diameter, $95 \mathrm{~mm}$ in length, and weighs $57 \mathrm{~g}$ (see Fig. 7).

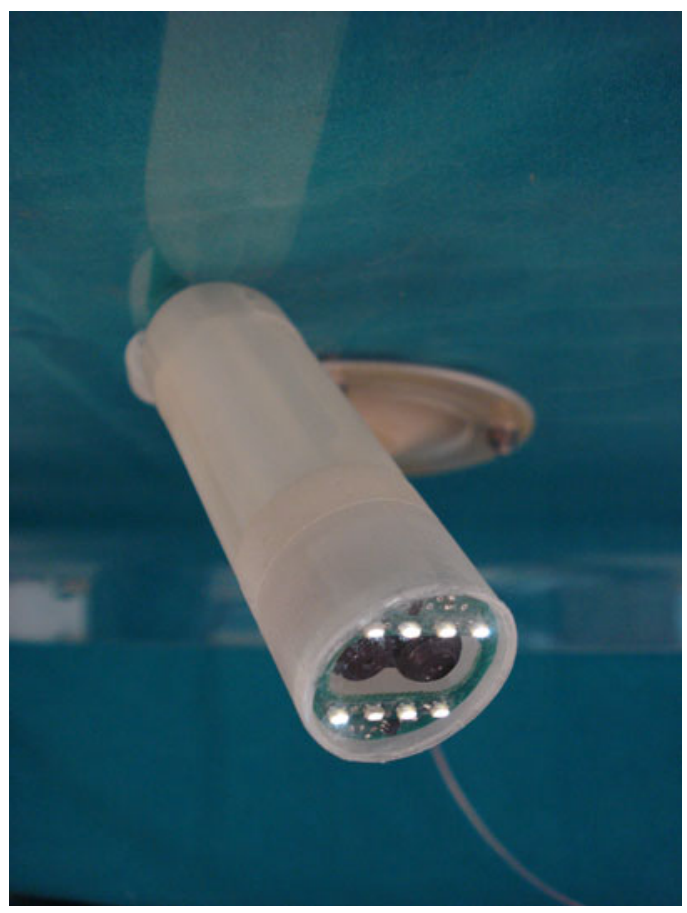

Fig. 7. Assembled prototype magnetically anchored to the abdomen simulator.

\section{Tilt Simple Model Description and EPMs Selection}

Considering an average abdominal wall thickness of $30 \mathrm{~mm}$ [47], the magnetic attraction force should be adequate to lift the robot against its own weight and to guarantee MIM functionality, satisfying the following simple static relations:

$$
\begin{aligned}
& F_{m}>F_{w} \\
& T_{m}>T_{\text {mim }} \\
& T_{\text {mot }}>T_{\text {mim }}
\end{aligned}
$$

where $F_{m}$ and $F_{w}$ are the EPMs-IPM magnetic attraction force and the device weight force, respectively. $T_{m}$ is the magnetic torque exerted by the EPMs on the IPM, $T_{\mathrm{mim}}$ is the maximum torque exerted by the weight of the device on the IPM pivot and $T_{\text {mot }}$ is the total torque that the brushless motor transmits to the IPM by means of the internal mechanism (see Fig. 1). Due to the very slippery properties of the abdomen wall, the friction between tissue and camera robot was neglected at this stage.

The camera device weighs $57 \mathrm{~g}$, therefore, a magnetic attraction force larger than $560 \mathrm{mN}\left(F_{w}\right)$ is required to completely lift the robot at a distance of $30 \mathrm{~mm}$. Given mass and arrangement of all device components, its center of mass $(26 \mathrm{~mm}$ from the IPM pivot) was derived and $T_{\mathrm{mim}}$ was analytically calculated as $14.76 \mathrm{mNm}$. Finally, as previously derived, $T_{\mathrm{mot}}$ is $445 \mathrm{mNm}$.

Since the EPMs must be easily handled by the doctor, and on the basis also of our simple model, two off-the-shelf (KJ Magnetics, Jamison, PA) cubic $(25.5 \mathrm{~mm} \times 25.5 \mathrm{~mm} \times 25.5 \mathrm{~mm})$ magnets (NdFeB, N52) were selected as best compromise between external magnetic field maximization and size. FEM analysis of these permanents magnets was performed to predict magnetic forces $F_{m}$ and torques $T_{m}$ (see Fig. 8). 


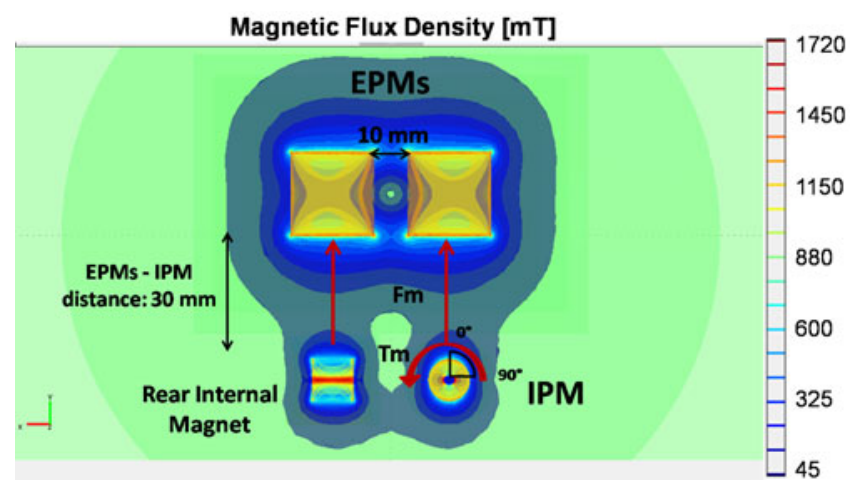

Fig. 8. FEM simulation of interaction between the magnets. The selected mesh consisted in about 1350000 elements, with a minimum quality ratio of 0.35 .

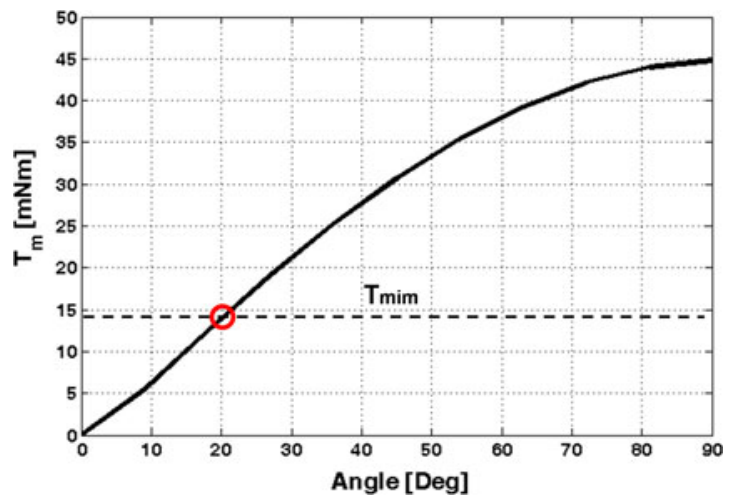

Fig. 9. Plot of the $T_{m}$ on the IPM as function of the rotation angle. The circle highlights when the $T_{m}$ is equal to $T_{\mathrm{mim}}$.

The magnetic attraction force between EPMs and IPM $F_{m}$ is $2.8 \mathrm{~N}$, while the magnetic torque $T_{m}$ goes from 0 to $45 \mathrm{mNm}$ for an IPM rotation angle ranging from $0^{\circ}$ to $90^{\circ}$ as represented in Fig. 9. The $T_{\text {mim }}$ torque was reached for an IPM rotation angle of about $20^{\circ}$.

Still based on the simulation results, the maximum magnetic flux density that surrounded the two brushless motors may be evaluated as being equal to $0.1 \mathrm{~T}$, thus satisfying the negligible effect of the magnetic field on the actuator features.

Finally, the two EPMs were embedded into a plastic case to improve handling. In case of a thicker abdominal wall, larger EPMs can be used to cope with the increased distance.

\section{EXPERIMENTAL RESULTS}

\section{A. System Functionality Characterization}

After assembling the robot, a number of bench tests were carried out to evaluate system performance and reliability. First, anchoring, pan, and translation stability between EPMs and the camera system were verified by simply moving the robot by hand in a Plexiglas simulator abdomen for LESS, having a simulated wall thickness of $30 \mathrm{~mm}$. Then, the active DOFs were evaluated by controlling the span motion with the PC interface. As regards the tilt DOF, a $0-90^{\circ}$ magnet rotation range was set. A span of $70^{\circ}$ is about 1.5 seconds long, confirming the speed theoretically derived during the design phase; after about $70^{\circ}$, MIM is no longer effective. During the tilt motion, the link between the rear magnet and the robot hampered robot rotation, thus limiting the robot tilt range. A redefined design of the body-tank link, e.g., using a spring element, would allow this problem to be solved. Thanks to the PC interface, the embedded motors may be controlled step by step; consequently, the highest MIM resolution obtained was lower than $0.01^{\circ}$. Active roll DOF features were evaluated, still using the PC control interface. No problems occurred for the $\pm 90^{\circ}$ range that was performed in about $0.5 \mathrm{~s}$, thus confirming the horizon adjustment high speed, whereas the highest resolution obtained for the step motor control was lower than $0.01^{\circ}$ in this case also.

In order to evaluate the results obtained with the approximated model for lighting distribution, an experimental test was carried out. The system was placed in the laparoscopic simulator at increasing distances (i.e., from $50 \mathrm{~mm}$ to $150 \mathrm{~mm}$, with a $10 \mathrm{~mm}$ pitch) from a monochromatic flat target, acquiring a stereo pair for each distance. The obtained images were processed in order to evaluate the percentage of the FOV having a $\mathrm{CV}$ less than 5\%, which is the same parameter used in the model. The experimental distributions obtained confirm theoretical predictions (see Fig. 10). Since the lowest intensity value obtained is far from zero, the developed illumination system shows its effectiveness in overcoming the minimum intensity value required to make the cameras work correctly. However, as shown in Fig. 11, illumination uniformity is less than in the model results. This may be due to scattering and reflective phenomena that, although neglected in the model, increase lighting distribution slopes and so worsen illumination uniformity [41]. As the differences between theoretical and experimental FOV percentage have a maximum value below $0.06 \%$ and an average value of $0.045 \%$, this study validates the implemented model. Moreover, during the tests, our lighting system qualitatively guaranteed images with satisfactory brightness and without any illumination distribution discontinuities.

\section{B. Assessment of the System in Medical Tasks on Bench}

To obtain a quantitative evaluation of the prototype's operative performance, a comparative study between a standard 2-D laparoscope and our 3-D imaging robot was also carried out. Both systems were mounted in the plexiglas LESS simulator; 16 surgeons were asked to perform two basic tasks in two different abdomen quadrants, and execution time was recorded. The first task performed in the right lower quadrant consisted in inserting ten rings into ten needles using an LESS pincer (pick-and-place task), while the second task, conducted in the left upper quadrant, consisted in performing a single suture on a synthetic skin (suturing task). At the beginning of each task, the robot was located near the insertion port. It was then roughly moved on the target area by using the EPMs, and the exact point of view was reached thanks to the active MIM and roll adjustment. When the traditional laparoscope was used, the task was always started by positioning the laparoscope perpendicular to 


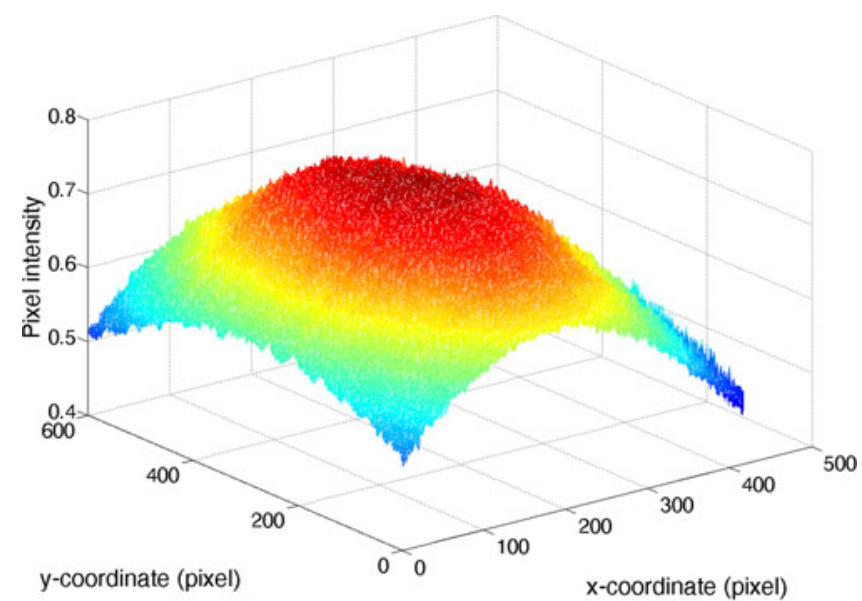

(a)

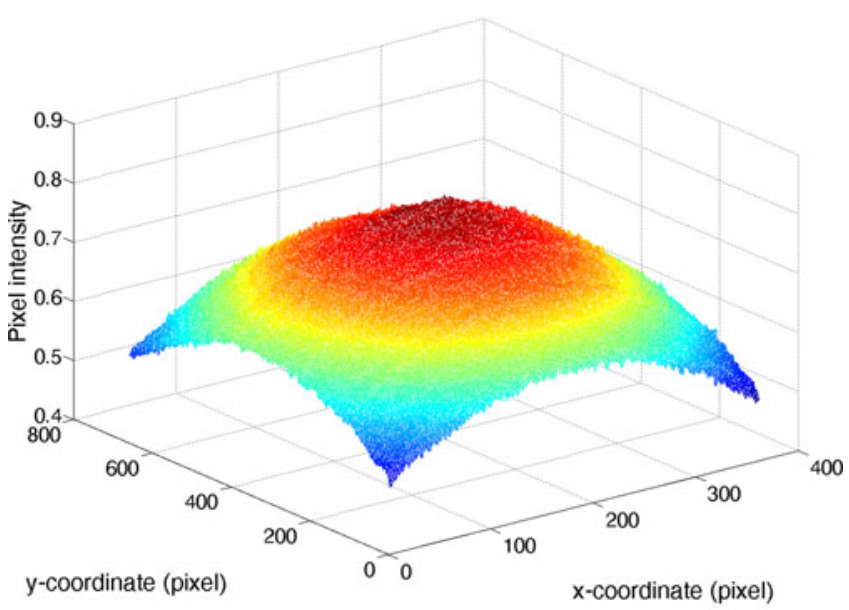

(b)

Fig. 10. Experimental lighting distribution obtained at (a) $50 \mathrm{~mm}$ in depth, and (b) $100 \mathrm{~mm}$ in depth. The $z$-axis is the pixel intensity of the acquired image of the target. As the processing unit embedded in the camera performs while balancing operation, the resulting dynamic range of the intensity versus depth is normalized. The $x$-axis and the $y$-axis are the image coordinates, measured in pixels.

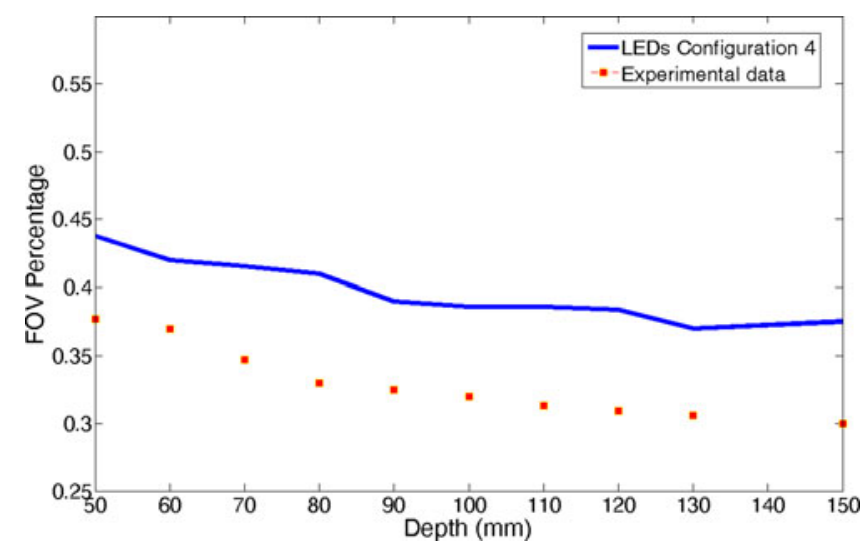

Fig. 11. Comparison between theoretical and experimental illumination uniformity. The evaluated parameter is still the FOV percentage, varying along the scene depth, having a CV less than 5\%. The blue line represents the theoretical result obtained using the LED configuration. The red dotted line represents the experimental results obtained by processing the acquired images of the target.
TABLE II

RESUlts OBTAINED DURING THE QUANTITATIVE COMPARISON TEST ON PERformance TIME

\begin{tabular}{|c|c|c|c|}
\hline & $\begin{array}{l}\text { Average } \\
\text { Execution } \\
\text { time 3D }\end{array}$ & $\begin{array}{l}\text { Average } \\
\text { Execution } \\
\text { time 2D }\end{array}$ & $\mathrm{p}$-value \\
\hline Pick and Place & $128 \mathrm{~s}$ & $144 \mathrm{~s}$ & 0.0011 \\
\hline Suturing & $105 \mathrm{~s}$ & $124 \mathrm{~s}$ & 0.0202 \\
\hline
\end{tabular}

the skin. After manual pointing by the surgeon, it was then held in the same position by an assistant.

In order to avoid learning bias, the order of the imaging system was randomized. Average execution times were evaluated using the analysis of variance (ANOVA) test, taking differences as statistically significant when $p \leq 0.05$ [48]. As summarized in Table II, average execution time was about $20 \mathrm{~s}$ lower in 3 -D viewing conditions for both tasks. Moreover, these large differences were statistically significant when using the ANOVA test.

This demonstrated that the developed stereoscopic imaging system significantly improves speed and efficiency in both low (pick and place) and high (suture) complexity tasks, thanks to a better relative distance and motion control. This helps to perform tasks more rapidly under 3-D viewing conditions, regardless of their complexity and of the doctor's individual surgical experiences [49], [50].

After the quantitative test, doctors were asked to answer a questionnaire in order to assess the quality of the stereo images provided by our prototype. Almost $80 \%$ of them greatly appreciated the 3-D imaging provided by our platform. On the other hand, some of them found it stressful to properly fuse the stereo images provided by the autostereoscopic display.

It is worth mentioning that results related to the 3-D display are valid only for the specific parallax barrier technology. Indeed, different technological solutions to achieve autostereoscopy could result in different performances in terms of userfriendliness and effectiveness [51].

However, all participants qualitatively confirmed the stereoscopic depth range from $50 \mathrm{~mm}$ to $150 \mathrm{~mm}$ and appreciated the brightness of the images. As to the robotic system, the surgeons' qualitative assessments of the device were good in terms of reliability, motion resolution, and simple control. Being able to reach appropriate and nonpreplanned points of view of different areas inside the abdominal cavity was considered to be the main advantage of the robotic prototype. The high number of DOFs (active and passive) of the system, which always ensure good mobility inside the abdomen, was a very appreciated quality. In particular, the use of manual operation for rough positioning and of robotic control for fine adjustment greatly enhanced camera pointing precision.

Finally, the roll DOF was considered fundamental in restoring the correct horizon position after robot motion, thus facilitating understanding of the scenario and, therefore, of the entire surgical procedure. 


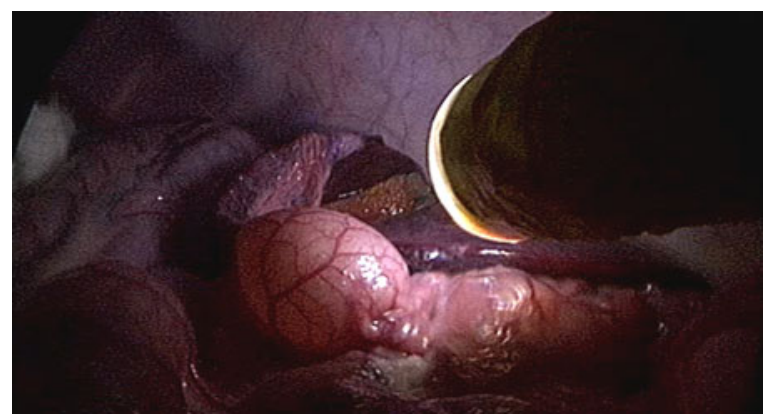

Fig. 12. Additional traditional laparoscope was inserted during the in vivo test to follow and evaluate the motion of the robot within the abdominal cavity. In the figure, the robot (on the right) is observing a section of the abdominal cavity (intestine and liver).

\section{Assessment of the System in In Vivo Conditions}

A preliminary in vivo test was performed on a $35-\mathrm{kg}$ female pig to evaluate the capabilities of the entire robotic platform in a real LESS surgical scenario. The aim was to visualize and recognize the main organs of the abdominal cavity, placed in different quadrants, from different viewpoints.

Ethylene oxide sterilization of the device was performed on the entire device before the in vivo test.

The experiment was conducted in an authorized laboratory with the assistance and collaboration of a specially trained medical team, in compliance with all ethical requirements and regulatory issues related to animal experiments. After intravenous sedation of the animal, an LESS procedure was performed using an SILS port (Covidien, Norwalk, CT).

The following procedure was executed for the insertion, use, and removal of the stereocamera robot.

1) The abdomen was incised at the navel.

2) The stereoscopic robotic camera was inserted in the abdomen.

3) The robot was magnetically anchored.

4) The flexible cable crossed the hole of the umbilical port from the side of the electronic connector $(D<10 \mathrm{~mm})$

5) The umbilical port was inserted in the pig's abdomen.

6) The abdomen was inflated.

7) The stereocamera robot was moved and actuated to focus on stomach, liver, spleen, intestine, colon, diaphragm, and gall bladder. Whenever, a change in the abdominal quadrant was required, the device was repositioned by magnetic dragging.

8) At the end of the procedure, the EPMs were removed.

9) The single-site port was retrieved with the robot through the abdominal wall incision.

The entire insertion procedure described earlier (steps 1-6) was easily performed. The prototype was correctly anchored to the abdomen wall. Pneumoperitoneum was established through the valve. Then, the robot was moved within the abdominal cavity. The robot successfully enabled a visual survey of the entire cavity (see Fig. 12).

All the organs were clearly identifiable. The illumination provided by the LEDs was found suitable for LESS by the surgeon and the 3-D perception on the autostereoscopic display allowed the entire surgical team to easily follow the procedure. During the inspection procedure, an endoscopist was able to operate both the EPMs and the button interface by following commands from the surgeon. The anchoring and rough motion of the robot with the EPMs was very stable, and the endoscopist found the other active DOFs very simple to control (step 7). Once the abdominal cavity visual inspection was completed, the removal procedure (steps 8-9) was successfully carried out without any problem. At the end of the in vivo test both the surgeon's and endoscopist's qualitative assessments of the device were very positive. As demonstrated also during the bench tests, the three passive DOFs always allow the desired abdominal area to be reached, whereas the two active DOFs (tilt and roll) ensure precise points of view and guarantee adequate speed, high resolution, and sufficient span angle.

\section{CONCLUSION}

An innovative vision platform for LESS, based on a magnetically activated stereoscopic wired robot, has been proposed in this paper. The presented softly tethered robot embeds a stereoscopic vision module, an LEDs lighting system, magnetic fixation to the abdomen wall and 5 DOFs (two active and three passive) for stereocamera steering. A working prototype was designed and fabricated $(25 \mathrm{~mm}$ in diameter, $95 \mathrm{~mm}$ in length, and $57 \mathrm{~g}$ in weight).

The robotic endoscope can be inserted through a $25-\mathrm{mm}$ incision and magnetically fixed on the abdominal wall. As in [13]-[18], a rough position can be obtained along the abdominal wall by dragging the robot by hand motion of the EPMs, thus allowing the endoscope to easily reach areas placed in different abdominal quadrants and so providing completely new points of view. Accordingly with other prototypes [13]-[18], the novel robotic endoscope has the potential to restore triangulation for the surgeon and to reduce both instrument collision and procedure invasiveness. Furthermore, the present device can provide a larger viewing volume than a traditional laparoscope that is restricted by the fulcrum point of insertion. Another main advantage offered by the robot is that the space in the access port is only partly taken up by a thin cable $(2 \mathrm{~mm})$, thus leaving space for the access of additional instrumentation that could be useful during complex surgical procedures.

In comparison with [13]-[18], once the stereocamera is positioned, fine tilt, and roll orientation can be obtained by exploiting the two active embedded mechanisms. Unlike [17]-[19], the $0-70^{\circ}$ tilt angle of view is provided by the active MIM and may be compared to the span obtained with a traditional endoscope during LESS surgical procedures. Additionally, since the tilting motion is not manual as in [13]-[16], but motorized, image stability, and motion resolution are greatly enhanced. Again compared to standard laparoscopes, the proposed system has a robotized rotational DOF around its long axis, thus allowing the image to be rotated. We restored this feature, which is lacking in all other robotic cameras [12]-[20], with a dedicated gear mechanism. Consequently, the roll active embedded motion always guarantees correct horizon adjustment with a span of $\pm 90^{\circ}$ (see Fig. 13). The speed and resolution of the two 


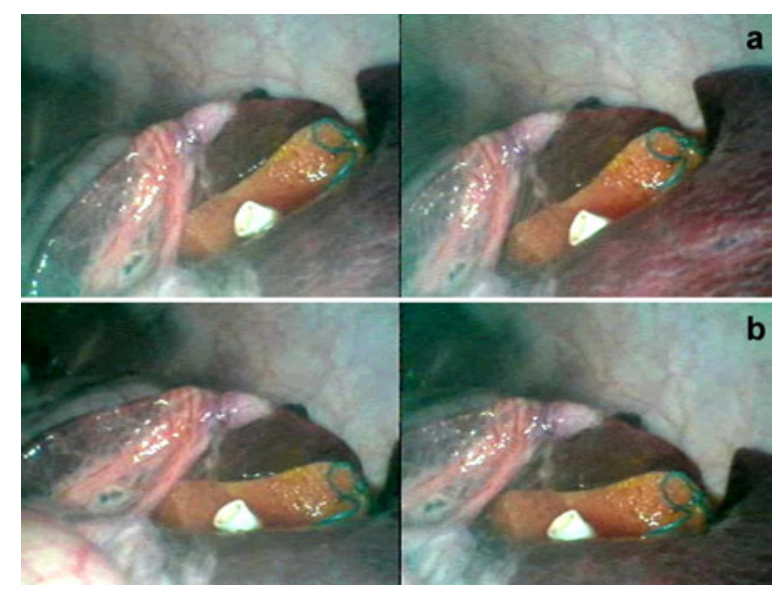

Fig. 13. Stereoscopic robotic point of view during visual inspection in the abdominal cavity. The left and right sides represent the two images from the stereocamera. (a) Images directly taken by the cameras when the robot is placed on the abdominal wall. (b) Images taken after horizon adjustment thanks to the active roll mechanism.

motorized DOFs is sufficient to ensure quick adjustment and reliable motion. Considering [20], the embedded illumination system and the possible magnetic adjustment along the abdomen wall, combined with additionally and highly precise internal DOFs, represent the main improvements brought about.

Regarding the qualitative-assessment tests carried out, physicians positively evaluated the stereoscopic effect, the brightness of the images and the friendliness of the 5 DOFs steering mechanism. Compared to standard LESS imaging systems, they also appreciated the FOV provided and the possibility to precisely tilt and rotate the imaging point of view, thus balancing the lower FOV compared to standard endoscopes [7]. Qualitatively, the imagery was sufficient to conclude the in vivo abdominal cavity visual inspection. As demonstrated by the comparison test, the stereoscopic effect showed good results in terms of binocular cues and content handiness. 3-D image stream allowed better performances as regards efficiency and execution speed, compared to conventional 2-D vision in both low and high complexity tasks. The autostereoscopic display allowed all participants to perceive the third dimension, consequently, the surgeon was not isolated from the surgical scenario and the assistants were always aware of the status of the procedure. Depth perception and relative motion perception guaranteed by binocular cues were appreciated. $80 \%$ of participants were in fact mostly made up of persons who found it effortless and natural to enter into the third dimension provided by the autostereoscopic screen. The development of new-generation autostereoscopic displays may further improve this condition.

Future developments intend improving camera resolution in order to enhance image quality. Moreover, a new processing unit will need to be developed to improve noise filtering and image sharpness. Finally, a future smaller prototype will be considered in order to introduce the entire robot through a traditional surgical trocar $(\phi=12 \mathrm{~mm})$, thus expanding the impact on standard laparoscopic procedures.

\section{ACKNOWLEDGMENT}

The authors would like to thank N. Funaro for manufacturing the prototypes. They would also like to thank G. Sardi for his technical support on the FEM simulation and G. Petroni for his technical support on PC and button control interface.

\section{REFERENCES}

[1] C. R. Tracy, J. D. Raman, J. A. Cadeddu, and A. Rane, "Laparoendoscopic single-site surgery in urology: Where have we been and where are we heading?" Nat. Clin. Pract. Urol., vol. 5, pp. 561-568, 2008.

[2] W. Brunner, J. Schirnhofer, N. Waldstein-Wartenberg, R. Frass, K. Pimpl, and H. Weiss, "New: Single-incision transumbilical laparoscopic surgery," Eur. Surg., vol. 41, pp. 98-103, 2009.

[3] M. Neto, A. Ramos, and J. Campos, "Single port laparoscopic access surgery," Tech. Gastrointestinal Endosc., vol. 11, pp. 84-93, 2009.

[4] Peter Weibl, "Current limitations and perspectives in single port surgery: Pros and cons laparo-endoscopic single-site surgery (LESS) for renal surgery," Diagn. Ther. Endosc., vol. 2010, pp. 759431-1-759431-3, 2010.

[5] I. S. Gill, A. P. Advincula, M. Aron, J. Caddedu, D. Canes, P. G. Curcillo, M. M. Desai, J. C. Evanko, T. Falcone, V. Fazio et al., "Consensus statement of the consortium for laparoendoscopic single-site surgery," Surg. Endosc., vol. 24, pp. 762-768, 2010.

[6] S. S. Kommu and A. Rané, "Devices for laparo-endoscopic single-site surgery in urology," Exp. Rev. Med. Devices, vol. 6, pp. 95-103, 2009.

[7] "Karl Storz official web site" (Oct. 11, 2010). [Online]. Available: http://www.karlstorz.com/

[8] "Olympus KeyMed official web site" (Oct. 9, 2010). [Online]. Available: http://www.olympuskeymed.com/

[9] J. H. Kaouk, R. K. Goel, G. P. Haber, S. Crouzet, and R. J. Stein, "Robotic single-port transumbilical surgery in humans: Initial report," BJU Int., vol. 103, pp. 366-369, 2008.

[10] R. J. Stein, W. M. White, R. K. Goel, B. H. Irwin, G. P. Haber, and J. H. Kaouk, "Robotic laparoendoscopic single-site surgery using GelPort as the access platform," Eur. Urol., vol. 57, pp. 132-137, 2010.

[11] T. Hu, P. K. Allen, N. J. Hogle, and D. L. Fowler, "Insertable surgical imaging device with pan, tilt, zoom, and lighting," Int. J. Robot., vol. 28, pp. 1373-1386, 2009.

[12] T. Kawahara, T. Takaki, I. Ishii, and M. Okajima, "Development of a broad-view camera system for minimally invasive surgery," presented at the IEEE/RSJ Int. Conf. on Intelligent Robots and Systems., Taipei, Taiwan, Oct. 18-22, 2010.

[13] I. S. Zeltser, R. Bergs, R. Fernandez, L. Baker, R. Eberhart, and J. A. Cadeddu, "Single trocar laparoscopic nephrectomy using magnetic anchoring and guidance system in the porcine model," J. Urol., vol. 178, pp. 288-291, 2007.

[14] J. A. Cadeddu, R. Fernandez, M. Desai, R. Bergs, C. Tracy, S. J. Tang, P. Rao, M. Desai, and D. Scott, "Novel magnetically guided intraabdominal camera to facilitate laparoendoscopic single-site surgery: Initial human experience," Surg. Endosc., vol. 23, pp. 1894-1899, 2009.

[15] M. Fakhry, B. Gallagher, F. Bello, and G. B. Hanna, "Visual exposure using single-handed magnet-driven intra-abdominal wireless camera in minimal access surgery is better than $30^{\circ}$ endoscope," Surg. Endosc., vol. 23, pp. 539-543, 2009.

[16] P. Swain, R. Austin, K. Bally, and R. Trusty, "Development and testing of a tethered, independent camera for notes and single-site laparoscopic procedures," Surg. Endosc., vol. 24, pp. 2013-2021, 2010.

[17] A. C. Lehman, K. A. Berg, J. Dumpert, N. A. Wood, A. Q. Visty, M. E. Rentschler, S. R. Platt, S. M. Farritor, and D. Oleynikov, "Surgery with cooperative robots," Comput. Aided Surg., vol. 13, pp. 95-105, 2008

[18] S. R. Platt, J. A. Hawks, and M. E. Rentschler, "Vision and task assistance using modular wireless in vivo surgical robots," IEEE Trans. Biomed. Eng., vol. 56, no. 6, pp. 1700-1710, Jun. 2009.

[19] T. Hu, P. K. Allen, T. Nadkarni, N. J. Hogle, and D. L. Fowler, "Insertable stereoscopic 3D surgical imaging device with pan and tilt," in Proc. 2nd IEEE RAS/EMBS Int. Conf. Biomed. Robot. Biomechatronics, Scottsdale, AZ, Oct. 19-22, 2008, pp. 311-316.

[20] D. L. Fowler, T. H. T. Nadkarni, P. K. Allen, and N. J. Hogle, "Initia trial of a stereoscopic, insertable, remotely controlled camera for minimal access surgery," Surg. Endosc., vol. 24, pp. 9-15, 2010.

[21] M. Silvestri, M. Simi, C. Cavallotti, M. Vatteroni, P. Valdastri, A. Menciassi, and P. Dario, "Design of a magnetically stereoscopic 
system for single port laparoscopy," in Proc. 3rd Hamlyn Symp. Med. Robot., London, U.K., May 25, 2010, pp. 63-64.

[22] M. Vatteroni, D. Covi, C. Cavallotti, L. Clemente, P. Valdastri, A. Menciassi, P. Dario, and A. Sartori, "Smart optical CMOS sensor for endoluminal application," Sens. Actuators A, Phys., vol. 162, no. 2, pp. 297-303, 2010.

[23] J. R. Romanelli and D. B. Earle, "Single-port laparoscopic surgery: An overview," Surg. Endosc., vol. 23, pp. 1419-1427, 2009.

[24] C. M. Grossmann, "A new AS-display as part of the MIRO lightweight robot for surgical applications," Proc. SPIE, Int. Soc. Opt. Eng., vol. 7524, pp. 752403-1-752403-12, 2010

[25] M. Silvestri, M. Simi, C. Cavallotti, M. Vatteroni, V. Ferrari, C. Freschi, P. Valdastri, A. Menciassi, and P. Dario, "Autostereoscopic threedimensional viewer evaluation through comparison with conventional interfaces in laparoscopic surgery," Surg. Innovation, vol. 18, pp. 223-230, 2011.

[26] UDA Mueller-Richter, A. Limberger, P. Weber, W. Spitzer, and M. Schilling, "Comparison between three-dimensional presentation of endoscopic procedures with polarization glasses and an autostereoscopic display," Surg. Endosc., vol. 17, pp. 502-504, 2003.

[27] Z. Y. Alpaslan, S. C. Yeh, A. A. Rizzo, and A. A. Sawchuk, "Quantitative comparison of interaction with shutter glasses and autostereoscopic displays," Proc. SPIE, Int. Soc. Opt. Eng., vol. 5664, pp. 616-625, 2005.

[28] M. Harris, "3D without four eyes," IEEE Spectr., vol. 47, no. 12, pp. 5056, Dec. 2010

[29] "Microsoft official web site" (Oct. 11, 2010). [Online]. Available: http://www.microsoft.com/showcase/last access

[30] "Sharp official web site" (Oct. 25, 2010). [Online]. Available: http://www.sle.sharp.co.uk

[31] P. Valdastri, C. Quaglia, E. Buselli, A. Arezzo, N. Di Lorenzo, M. Morino, A. Menciassi, and P. Dario, "A magnetic internal mechanism for precise orientation of the camera in wireless endoluminal applications," Endoscopy, vol. 42, pp. 481-486, 2010.

[32] M. Simi, G. Ciuti, S. Tognarelli, P. Valdastri, A. Menciassi, and P. Dario, "Magnetic link design for a robotic laparoscopic camera," J. Appl. Phys., vol. 107, no. 09B302, pp. 1-3, 2010.

[33] M. Ferrè, R. Aracil, and M. Sanchez-Uran, "Stereoscopic human interfaces," IEEE Robot. Autom. Mag., vol. 15, no. 4, pp. 50-57, Dec. 2008.

[34] J. H. Palep, "Robotic assisted minimally invasive surgery," J. Minimal Access Surg., vol. 5, pp. 1-7, 2009.

[35] C. Y. Chen, T. T. Yang, and W. S. Sun, "Optics system design applying a micro-prism array of a single lens stereo pair," Opt. Express, vol. 16, pp. 15495-15505, 2008.

[36] L. Hill and A. Jacobs, "3-D liquid crystal displays and their applications," Proc. IEEE, vol. 94, no. 3, pp. 575-589, Mar. 2006.

[37] "Miracube official web site" (Oct. 25, 2010). [Online]. Available: http://www.miracube3d.com

[38] A. Woods, T. Docherty, and R. Koch, "Image distortions in stereoscopic video systems," in Proc. SPIE, Stereoscopic Disp. Appl. IV, vol. 1915, San Jose, CA, 1993, pp. 36-48.

[39] N. Holliman, "Mapping perceived depth to regions of interest in stereoscopic images," in Proc. SPIE, Stereoscopic Disp. Virtual Reality Syst. XI, 2004, vol. 5291, pp. 1-12.

[40] "Nichia official web site" (Oct. 11, 2010). [Online]. Available: http://www.nichia.com. last access

[41] C. P. Poole, The Physics Handbook. Hoboken, NJ: Wiley-Interscience, pp. $188-202$.

[42] "Maxon official web site" (Oct. 30, 2010). [Online]. Available: http://www.maxon.net/

[43] M. Simi, P. Valdastri, C. Quaglia, A. Menciassi, and P. Dario, "Design, fabrication and testing of a capsule with hybrid locomotion for gastrointestinal tract exploration," IEEE/ASME Trans. Mechatronics, vol. 15, no. 2, pp. 170-180, Apr. 2010.

[44] "Roymech, mechanical engineering information web site" (Oct. 30, 2010). [Online]. Available: http://www.roymech.co.uk/Useful_ Tables/Drive/Gears.html

[45] P. Valdastri, R. J. Webster III, C. Quaglia, M. Quirini, A. Menciassi, and P. Dario, "A new mechanism for meso-scale legged locomotion in compliant tubular environments," IEEE Trans. Robot., vol. 25, no. 5, pp. 1047-1057, Oct. 2009.

[46] M. Quirini, A. Menciassi, S. Scapellato, C. Stefanini, and P. Dario, "Designand fabrication of a motor legged capsule for the active exploration of the gastrointestinal tract," IEEE/ASME Trans. Mechatronics, vol. 13, no. 2, pp. 169-179, Apr. 2008.
[47] C. Song, A. Alijani, T. Frank, G. B. Hanna, and A. Cuschieri, "Mechanical properties of the human abdominal wall measured in vivo during insufflation for laparoscopic surgery," in Surg. Endosc., 2006, vol. 20, pp. $987-990$.

[48] D. Freedman, R. Pisani, and R. Purves, Statistics. 4th ed. New York: Norton, 1991.

[49] S. H. Kong, B. M. Oh, H. Yoon, H. S. Ahn, H. J. Lee, S. G. Chung, N. Shiraishi, S. Kitano, and H. K. Yang, "Comparison of two- and threedimensional camera systems in laparoscopic performance: A novel 3D system with one camera," Surg. Endosc., vol. 24, pp. 1132-1143, 2010.

[50] V. Falk, J. Grunenfelder, J. I. Fann, and T. A. Burdon, "Influence of three-dimensional vision on surgical telemanipulator performance," Surg. Endosc., vol. 15, pp. 1282-1288, 2001.

[51] M. Barkowsky and P. Le Callet, "The influence of autostereoscopic 3D displays on subsequent task performance," in Proc. Stereoscopic Disp. Appl. XXI, vol. 7524, San Jose, CA, art. 752416, 2010.

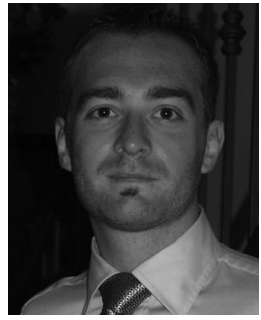

Massimiliano Simi received the Master's degree in biomedical engineering from the University of Pisa, Pisa, Italy, in April 2009. He is currently working toward the Ph.D. degree in biorobotics at The BioRobotics Institute, Scuola Superiore Sant'Anna, Pisa, Italy.

$\mathrm{He}$ has been with the Center for Research in Microengineering (CRIM) Laboratory as a Research Assistant since June 2008. His current research interests include medical robotics and biomechatronics.

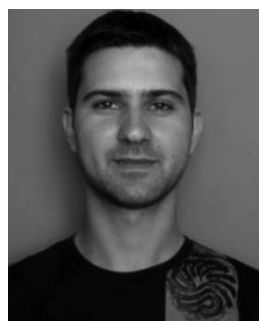

Michele Silvestri received the Master's degree in biomedical engineering from the University of Pisa, Pisa, Italy, in June 2010. He is currently working toward the Ph.D. degree in biorobotics at The BioRobotics Institute, Scuola Superiore Sant'Anna, Pisa.

His current research interests include biomechatronics and medical imaging.

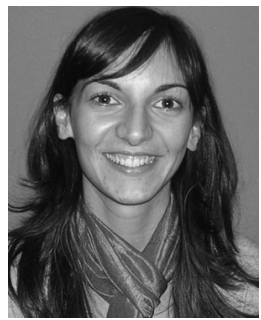

Carmela Cavallotti received the Master's degree in biomedical engineering (Hons.) from the Campus Bio-Medico University, Rome, Italy, in December 2007. She is currently working toward the Ph.D. degree in biobiorobotics at The BioRobotics Institute of the Scuola Superiore Sant'Anna, Pisa, Italy.

Her main research interests include the fields of vision systems for biomedical applications.

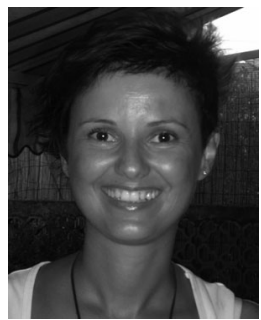

Monica Vatteroni was born in La Spezia, Italy, in 1975. She received the M.S. degree in electrical engineering from the University of Pisa, Pisa, Italy, in 2001, and the Ph.D. degree in physics from the University of Trento, Trento, Italy, in 2008.

She is currently with the Scuola Superiore Sant'Anna, Pisa, Italy as a Postdoctoral Fellow, where she is responsible for research and development of image sensors and vision systems. 


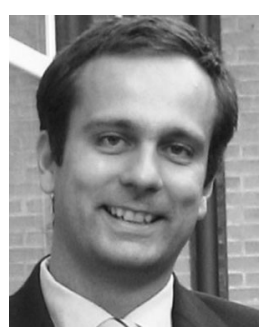

Pietro Valdastri (M'05) received the Master's (Hons.) degree in electronic engineering from the University of Pisa, Pisa, Italy, in 2002, and the Ph.D. degree in bioengineering from the Scuola Superiore Sant'Anna, Pisa.

He is currently an Assistant Professor at Vanderbilt University, Nashville, TN, and the Director of the STORM Lab.

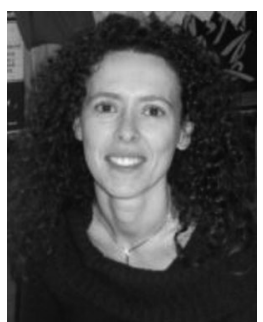

Arianna Menciassi (M'00) received the Master's degree in physics from the University of Pisa, Pisa, Italy, in 1995, and the Ph.D. degree in biomedical engineering from the Scuola Superiore Sant'Anna, Pisa, in 1999.

She is currently an Associate Professor of biomedical robotics at the Scuola Superiore Sant'Anna.

Dr. Menciassi serves on the Editorial Board of the IEEE/ASME TRANSACTION. OF MECHATRONICS.

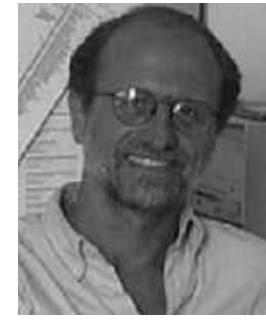

Paolo Dario (M'99-SM'01-F'03) received the Master's degree in mechanical engineering from the University of Pisa, Pisa, Italy, in 1977.

He is currently a Professor of biomedical robotics at the Scuola Superiore Sant'Anna, Pisa, where he supervises a team of about 150 young researchers. $\mathrm{He}$ has authored or coauthored more than 200 ISI journal papers, many international patents, and several book chapters on medical robotics. 